\title{
ESTIMATION OF DENSITY AND DISTRIBUTION FUNCTIONS OF A BURR X DISTRIBUTION
}

\author{
AmUlya Kumar Mahto \\ Department of Mathematics, Indian Institute of Technology Patna, Bihta-801106, India \\ Email: amulya.pma15@iitp.ac.in \\ YOGESH MANI TRIPATHI ${ }^{\star}$ \\ Department of Mathematics, Indian Institute of Technology Patna, Bihta-801106, India \\ Email:yogesh@iitp.ac.in \\ SANKU DEY \\ Department of Statistics, St. Anthonys College, Shillong-793001, Meghalaya, India \\ Email:sankud66@gmail.com
}

\begin{abstract}
SUMMARY
Burr type $\mathrm{X}$ distribution is one of the members of the Burr family which was originally derived by Burr (1942) and can be used quite effectively in modelling strength data and also general lifetime data. In this article, we consider efficient estimation of the probability density function (PDF) and cumulative distribution function (CDF) of Burr X distribution. Eight different estimation methods namely maximum likelihood estimation, uniformly minimum variance unbiased estimation, least square estimation, weighted least square estimation, percentile estimation, maximum product estimation, Cremér-von-Mises estimation and Anderson-Darling estimation are considered. Analytic expressions for bias and mean squared error are derived. Monte Carlo simulations are performed to compare the performances of the proposed methods of estimation for both small and large samples. Finally, a real data set has been analyzed for illustrative purposes.
\end{abstract}

Keywords and phrases: Maximum likelihood estimator, Uniformly minimum variance unbiased estimator.

AMS Classification: $62 \mathrm{~F} 10$

\section{Introduction}

Burr (1942) introduced twelve different forms of cumulative distribution functions for modeling lifetime data or survival data. Of these twelve distribution functions, Burr type $\mathrm{X}$ and Burr type XII were extensively used by the researchers. The cumulative distribution function of a Burr X distribution as proposed by Surles and Padgett (2001) is given by

$$
F(x ; \alpha, \gamma)=\left(1-e^{-(\gamma x)^{2}}\right)^{\alpha}, \quad x>0, \alpha>0, \gamma>0,
$$

\footnotetext{
* Corresponding author
}

(c) Institute of Statistical Research and Training (ISRT), University of Dhaka, Dhaka 1000, Bangladesh. 
and corresponding probability density function is given by

$$
f(x ; \alpha, \gamma)=2 \alpha \gamma^{2} x e^{-(\gamma x)^{2}}\left(1-e^{-(\gamma x)^{2}}\right)^{\alpha-1}, \quad x>0, \alpha>0, \gamma>0
$$

where $\alpha$ and $\gamma$ denote the shape and the scale parameters respectively. This distribution is also known as exponentiated Rayleigh or generalized Rayleigh distribution. We denote this distribution as $\operatorname{Burr} X(\alpha, \gamma)$ through out this article. It is observed that the two-parameter $\operatorname{Burr} X(\alpha, \gamma)$ distribution has several properties which are quite common to the two-parameter gamma, Weibull and generalized exponential (GE) distributions. The one parameter Burr X distribution (with $\gamma=1$ ) received maximum attention in manay works (Sartawi and Abu-Salih, 1991; Ahmad et al., 1997; Raqab, 1998; Surles and Padgett, 1998, 2001). In the recent past, Raqab and Kundu (2006) observed several interesting properties of Burr X distribution in their study and established relations with gamma, Weibull, exponentiated exponential and exponentiated Weibull distributions. The distribution function and the density function of $\operatorname{Burr} X(\alpha, \gamma)$ distribution have closed form. Due to this feature, it can be used very conveniently even for censored data. Unlike, gamma, Weibull and GE distributions it can have non-monotone hazard function, which can be very useful in many practical applications.

Raqab and Kundu (2006) observed that for $\alpha \leq 1 / 2$ Burr type $\mathrm{X}$ density is a decreasing function and it is a right skewed unimodal function for $\alpha>1 / 2$. They also observed that failure rates have the different shapes depending on the value of $\alpha$. For $\alpha \leq 1 / 2$, it has increasing failure rate and for $\alpha>1 / 2$, it is bathtub shaped. For more detailed properties of the Burr type $\mathrm{X}$ distribution (see Surles and Padgett, 2005).

It is a common practice with statisticians to focus on inferring the parameter(s) involved in the model. However, one would find it more useful to study the efficient estimation of the PDF and CDF instead of inferring the parameter(s) involved in the model. The problem of estimation of the PDF and the CDF is significant for many reasons. For example, PDF can be used for estimation of differential entropy, Renyi entropy, Kullback-Leibler divergence and Fisher information. For example, Nilsson and Kleijn (2007) considered the problem of estimating differential entropy using the data located on embedded manifolds. Authors mentioned that such studies have found widespread applications in various areas of signal processing such as source coding, pattern recognition and blind source separation, among others. Hampel (2008) discussed applications of entropy estimation in neuroscience. The concept of differential entropy can be used to infer random changes in neuron behavior under various experimental scenarios. Recently Mielniczuk and Wojtys (2010) considered estimation of Fisher information for a probability density supported on finite interval. These applications suggest that estimation of density function is an important problem in literature. Similarly CDF can be used for estimation of cumulative residual entropy, the quantile function, Bonferroni curve, Lorenz curve and both pdf and cdf can be used for estimation of probability weighted moments, hazard rate function, mean deviation about mean etc. For instance, Bratpvrbajgyran (2012) considered the problem of estimating cumulative residual entropy for the Rayleigh distribution. Aucoin et al. (2012) considered estimation of quantile of a two-parameter kappa distribution using different methods. A flood data set is analyzed in support of proposed statistical procedures and useful discussions are presented based on this numerical study. Longford (2012) further derived estimators 
of quantiles of normal, log-normal and Pareto distributions. The author studied a financial data on monthly returns and concluded that proposed estimators work quite well in such situations. In this paper, our focus is to obtain biased and unbiased estimators of the PDF and CDF using different classical methods of estimation.

Numerous statistical developments and applications of the $\operatorname{Burr} X(\alpha, \gamma)$ distribution has generated great interest among applied statisticians to study the efficient estimation of the PDF and the $\mathrm{CDF}$ of the $\operatorname{Burr} X(\alpha, \gamma)$ distribution. We consider several estimation methods: maximum likelihood estimation (MLE), uniformly minimum variance unbiased estimation (UMVUE), least square (LS) estimation and weighted least square (WLS) estimation, percentile estimation (PC), maximum product spacing estimation (MPS), Cramér-von-Mises (CVM) method of estimation and Anderson-Darling (AD) method of estimation and thereby aim to develop a guideline to choose the best estimation method for the $\operatorname{Burr} X(\alpha, \gamma)$ distribution. Similar kind of studies has appeared in the recent literature for other distributions (see Asrabadi, 1990; Dixit and Jabbari Nooghabi, 2010; Jabbari Nooghabi and Jabbari Nooghabi, 2010; Dixit and Jabbari Nooghabi, 2011; Bagheri et al., 2014, 2016, and the references cited therein).

Throughout this paper (except for Section 3), we assume $\alpha$ is unknown, but $\gamma$ is known. A future work is to extend the results of the paper to the case that all two parameters are unknown. In the literature one would find several papers where the PDF and the CDF have been estimated when all their parameters are unknown. For example, Duval (2013) investigated the nonparametric estimation of the jump density of a compound Poisson process from the discrete observation, Durot et al. (2013) obtained least-squares estimator of a convex discrete distribution, $\operatorname{Er}(1998)$ evaluated the unknown parameters in the polynomial using weighted residual method, Dattner and Reiser (2013) considered the estimation of distribution functions when data contains measurement errors and Przybilla et al. (2013) used maximum likelihood estimator to estimate the cumulative distribution function for the three-parameter Weibull CDF in presence of concurrent flaw populations.

Our present work is different from the existing work because we have considered eight methods of estimation for estimating pdf and cdf whereas in existing literature only five methods of estimation is considered to the best of our knowledge.

We have organized the rest of the content of this paper as follows. In Sections 2.1 and 2.2 we have derived MLEs and UMVUEs of density function and distribution function with their mean squared errors (MSEs) respectively. The LSEs and WLSEs are obtained in Section 2.3 and percentile estimation is discussed in Section 2.4. The other suggested methods of estimation are described in sections 2.5, 2.6 and 2.7 respectively. We have conducted a simulation study in Section 3 to assess the behavior of all estimators. We have analyzed a real data set for illustrative purpose in Section 4 . Finally, in Section 5, we conclude the paper. 


\section{Methods of Estimation}

\subsection{Maximum Likelihood Estimation}

In this section, we obtain MLEs of the PDF and the CDF of a $\operatorname{Burr} X(\alpha, \gamma)$ distribution. Suppose $X_{1}, X_{2}, \ldots, X_{n}$ denote independent and identically distributed random samples from the $\operatorname{Burr} X(\alpha, \gamma)$ distribution with known scale parameter $\gamma$. The likelihood function of $\alpha$ can be written as

$$
L(\alpha, x)=\prod_{i=1}^{n} 2 \alpha \gamma^{2} x_{i} e^{-\left(\gamma x_{i}\right)^{2}}\left(1-e^{-\left(\gamma x_{i}\right)^{2}}\right)^{\alpha-1},
$$

and the corresponding log-likelihood function is

$$
l=\ln L(x, \alpha) \propto n \ln (\alpha)+\sum_{i=1}^{n} \ln \left(x_{i}\right)-\gamma^{2} \sum_{i=1}^{n} x_{i}^{2}+(\alpha-1) \sum_{i=1}^{n} \ln \left(1-e^{-\left(\gamma x_{i}\right)^{2}}\right) .
$$

Considering the log likelihood function, we find the MLE of $\alpha$ as

$$
\hat{\alpha}=-\frac{n}{\sum_{i=1}^{n} \ln \left(1-e^{-\left(\gamma x_{i}\right)^{2}}\right)}=\frac{n}{T},
$$

where $T=-\sum_{i=1}^{n} \ln \left(1-e^{-\left(\gamma x_{i}\right)^{2}}\right)$. Now consider the transformation

$$
Y_{i}=g\left(X_{i}\right)=-\ln \left(1-e^{-\left(\gamma X_{i}\right)^{2}}\right) \text { with } g^{-1}\left(y_{i}\right)=(1 / \gamma)\left[-\ln \left(1-e^{y_{i}}\right)\right]^{1 / 2} .
$$

Then it is seen that probability density of $Y_{i}$ turns out to be,

$$
f_{Y_{i}}\left(y_{i}\right)=f_{X_{i}}\left(g^{-1}(x)\right)\left|\frac{d^{-1} g\left(x_{i}\right)}{d y_{i}}\right|=\alpha e^{-\alpha y_{i}}, y_{i}>0, \alpha>0 .
$$

Thus, we see that if $X$ has a $\operatorname{Burr} X(\alpha, \gamma)$ distribution then $Y_{i}=-\ln \left(1-e^{-\left(\gamma x_{i}\right)^{2}}\right)$ has an exponential distribution with rate $\alpha$, i.e. $Y_{i} \sim \exp (\alpha)$. As we know that the sum of independently and identically distributed exponential distribution follows a gamma distribution. Thus $T=$ $-\sum_{i=1}^{n} \ln \left(1-e^{-\left(\gamma x_{i}\right)^{2}}\right)$ follows gamma $G(n, \alpha)$ distribution having density function as

$$
f_{T}(t)=\frac{\alpha^{n}}{\Gamma n} t^{n-1} e^{-\alpha t}, t>0 .
$$

Further, we find that the MLE $\hat{\alpha}=(n / T)=W$ has an inverse gamma $I G(n, \alpha n)$ distribution having density function as

$$
f_{W}(w)=\frac{(n \alpha)^{n}}{\Gamma n}(1 / w)^{n+1} e^{-\alpha n / w}, w>0 .
$$

Note that $E(\hat{\alpha})=n \alpha /(n-1)$ and thus the MLE $\hat{\alpha}$ is a biased but consistent estimator of the parameter $\alpha$. The invariance property of maximum likelihood method is applied and the desired estimators of PDF and CDF are then obtained as

$$
\hat{f}(x)=2 \hat{\alpha} \gamma^{2} x e^{-(\gamma x)^{2}}\left(1-e^{-(\gamma x)^{2}}\right)^{\hat{\alpha}-1} \text { and } \hat{F}(x)=\left(1-e^{-(\gamma x)^{2}}\right)^{\hat{\alpha}} .
$$


Here we show that the estimators $\hat{f}(x)$ and $\hat{F}(x)$ are biased for the PDF $f(x)$ and the CDF $F(x)$ respectively and also obtain their mean square errors. For presentation and calculation simplicity, we rewrite $\hat{f}(x)$ and $\hat{F}(x)$ as

$$
\hat{f}(x)=2 w x \eta(\xi)^{w-1} \text { and } \hat{F}(x)=(\xi)^{w},
$$

where $\eta=\gamma^{2} e^{-(\gamma x)^{2}}$ and $\xi=1-e^{-(\gamma x)^{2}}$. Before we obtain the mean squared errors, the following expectations are required. We have

$$
\begin{aligned}
E(\hat{f}(x))^{m} & =\int_{0}^{\infty}\left[2 w x \eta(\xi)^{w-1}\right]^{m} \frac{(n \alpha)^{n}}{\Gamma n}(1 / w)^{n+1} e^{-\frac{n \alpha}{w}} d w \\
& =(2 x \eta / \xi)^{m} \frac{(n \alpha)^{n}}{\Gamma n} \int_{0}^{\infty} w^{m-n-1} e^{-w m \ln (1 / \xi)-\frac{n \alpha}{w}} d w \\
& =2(2 x \eta / \xi)^{m} \frac{(n \alpha)^{(m+n) / 2}}{\Gamma n}[m \ln (1 / \xi)]^{-(m-n) / 2} K_{m-n}(2 \sqrt{m n \alpha \ln (1 / \xi)}) \\
E(\hat{F}(x))^{m} & =\int_{0}^{\infty}\left(\xi^{w}\right)^{m} \frac{(n \alpha)^{n}}{\Gamma n}(1 / w)^{n+1} e^{-\frac{n \alpha}{w}} d w \\
& =\frac{(n \alpha)^{n}}{\Gamma n} \int_{0}^{\infty} w^{-n-1} e^{-w(m \ln (1 / \xi))-\frac{n \alpha}{w}} d w \\
& =\frac{2(n \alpha)^{\frac{n}{2}}}{\Gamma n}[m \ln (1 / \xi)]^{n / 2} K_{-n}(2 \sqrt{m n \alpha \ln (1 / \xi)}) .
\end{aligned}
$$

The last equality follows from the following identity

$$
\int_{0}^{\infty} x^{\nu-1} e^{-\frac{\mu}{x}-\eta x} d x=2(\mu / \eta)^{\frac{\nu}{2}} K_{\nu}(2 \sqrt{\mu \eta})
$$

where $K_{\nu}(\cdot)$ is modified Bessel function of the second kind of order $\nu$ (see also, Bagheri et al., 2014).

Theorem 1. Desired expectations are

$$
E\left[(\hat{f}(x))^{m}\right]=2(2 x \eta / \xi)^{m} \frac{(n \alpha)^{(m+n) / 2}}{\Gamma n}[m \ln (1 / \xi)]^{-(m-n) / 2} K_{m-n}(2 \sqrt{m n \alpha \ln (1 / \xi)})
$$

and

$$
E\left[(\hat{F}(x))^{m}\right]=2 \frac{(n \alpha)^{n / 2}}{\Gamma n}[m \ln (1 / \xi)]^{n / 2} K_{-n}(2 \sqrt{m n \alpha \ln (1 / \xi)}) .
$$

Theorem 2. The mean squared errors of $\hat{f}(x)$ and $\hat{F}(x)$ respectively are

$$
\begin{aligned}
M S E(\hat{f}(x))= & 8 x^{2}\left(\frac{\eta}{\xi}\right)^{2} \frac{(n \alpha)^{(n+2) / 2}}{\Gamma n}[2 \ln (1 / \xi)]^{-(2-n) / 2} K_{2-n}(2 \sqrt{2 n \alpha \ln (1 / \xi)})-8 x f(x) \\
& \times\left(\frac{\eta}{\xi}\right) \frac{(n \alpha)^{(n+1) / 2}}{\Gamma n}[\ln (1 / \xi)]^{-(1-n) / 2} K_{1-n}(2 \sqrt{n \alpha \ln (1 / \xi)})+f^{2}(x),
\end{aligned}
$$


and

$$
\begin{aligned}
M S E(\hat{F}(x))= & 2 \frac{(n \alpha)^{n / 2}}{\Gamma n}[2 \ln (1 / \xi)]^{n / 2} K_{-n}(2 \sqrt{2 n \alpha \ln (1 / \xi)})-4 F(x) \frac{(n \alpha)^{n / 2}}{\Gamma n}[\ln (1 / \xi)]^{n / 2} \\
& \times K_{-n}(2 \sqrt{n \alpha \ln (1 / \xi)})+F^{2}(x) .
\end{aligned}
$$

Proof. We have

$$
M S E(\hat{f}(x))=E(\hat{f}(x)-f(x))^{2}=E(\hat{f}(x))^{2}-2 f(x) E(\hat{f}(x))+f^{2}(x) .
$$

The required expectations in the above expression can be obtained easily by substituting appropriate choice of $m$ in Theorem 2 to obtain the desired MSE. Similarly we can obtain $M S E(\hat{F}(x))$.

\subsection{Uniformly Minimum Variance Unbiased Estimation}

In this section, our aim is to obtain UMVUEs of the PDF and the CDF of the specified distribution. We see that $T=-\sum_{i=1}^{n} \ln \left(1-e^{-\left(\gamma x_{i}\right)^{2}}\right)$ is complete and sufficient for estimating $\alpha$ for given $\gamma$ and $T$ follows a gamma $\mathrm{G}(n, \alpha)$ distribution. One may refer to Ferguson (1967) for this useful result. Following Lehmann-Scheffé theorem if $g\left(x_{1} \mid t\right)=h^{\star}(t)$ is the conditional PDF of $X_{1}$ given $T=t$. Then we have (see also, Bagheri et al., 2014),

$$
E\left[h^{\star}(T)\right]=\int g\left(x_{1} \mid t\right) f(t) d t=\int g\left(x_{1}, t\right) d t=f\left(x_{1}\right),
$$

where $g\left(x_{1}, t\right)$ denotes the joint PDF of $\left(X_{1}, T\right)$. Thus $h^{\star}(T)$ is the UMVUE of $f(x)$.

Lemma 2.1. The conditional distribution of $V$ given that $T=t$ is obtained as

$$
f_{V \mid T=t}(v \mid t)=\frac{(n-1)(t-v)^{n-2}}{t^{n-1}}, v<t<\infty
$$

where $V=-\ln \left(1-e^{-\left(\gamma x_{1}\right)^{2}}\right)$.

Proof. We have

$$
\begin{aligned}
f_{V \mid T=t}(v \mid t) & =\frac{f(t, v)}{f(t)} \\
& =\frac{f\left(v,-\sum_{i=2}^{n} \ln \left(1-e^{-\left(\gamma x_{i}\right)^{2}}\right)=t-v\right)}{f_{T}(t)} \\
& =\frac{\alpha e^{-\alpha v} \frac{\alpha^{n-1}}{\Gamma(n-1)}(t-v)^{n-2} e^{-\alpha(t-v)}}{\frac{\alpha^{n}}{\Gamma n} t^{n-1} e^{-\alpha t}} \\
& =\frac{(n-1)(t-v)^{n-2}}{t^{n-1}}, v<t<\infty .
\end{aligned}
$$

In the theorem stated below we give UMVUEs of $f(x)$ and $F(x)$. 
Theorem 3. The expression

$$
\hat{f}(x)=\frac{(n-1)\left(t+\ln \left(1-e^{-(\gamma x)^{2}}\right)\right)^{n-2}}{t^{n-1}} \times \frac{2 x \gamma^{2} e^{-(\gamma x)^{2}}}{\left(1-e^{-(\gamma x)^{2}}\right)}
$$

for $-\ln \left(1-e^{-(\gamma x)^{2}}\right)<t<\infty$ is the UMVUE of $f(x)$ and also the UMVUE of $F(x)$ is as

$$
\hat{F}(x)=\left\{1+(1 / t) \ln \left(1-e^{-(\gamma x)^{2}}\right)\right\}^{n-1} .
$$

Proof. The estimator $\hat{f}(x)$ is the UMVUE of $f(x)$ follows from the Lehmann-Scheffé theorem and the previous lemma. Also $\hat{F}(x)$ is the UMVUE of $F(x)$ follows from the fact that

$$
\frac{d \hat{F}(x)}{d x}=\frac{d}{d x}\left[\left\{1+(1 / t) \ln \left(1-e^{-(\gamma x)^{2}}\right)\right\}^{n-1}\right]=\hat{f}(x) .
$$

Further we proceed to obtain MSEs of these UMVUE estimators. For notational simplicity, we take $\Omega=-\ln \left(1-e^{-(\gamma x)^{2}}\right)$. First we obtain the following two expectations:

$$
\begin{aligned}
E(\hat{f}(x))^{m} & =\int_{\Omega}^{\infty}\left(\frac{(n-1)\left[t+\ln \left(1-e^{-(\gamma x)^{2}}\right)\right]^{n-2}}{t^{n-1}} \times \frac{2 x \gamma^{2} e^{-(\gamma x)^{2}}}{\left(1-e^{\left.-(\gamma x)^{2}\right)}\right.}\right)^{m} \frac{\alpha^{n}}{\Gamma n} t^{n-1} e^{-\alpha t} d t \\
& =[(n-1) 2 x \eta / \xi]^{m} \frac{\alpha^{n}}{\Gamma n} \int_{\Omega}^{\infty} t^{n-m-1} e^{-\alpha t} \sum_{i=0}^{n}\left(\begin{array}{c}
m(n-2) \\
i
\end{array}\right)[(1 / t) \ln \xi]^{i} d t \\
& =[(n-1) 2 x \eta / \xi]^{m} \frac{\alpha^{n}}{\Gamma n} \sum_{i=0}^{n}\left(\begin{array}{c}
m(n-2) \\
i
\end{array}\right)(\ln \xi)^{i} \int_{\Omega}^{\infty} t^{n-m-i-1} e^{-\alpha t} d t \\
& =[(n-1) 2 x \eta / \xi]^{m} \frac{\alpha^{n}}{\Gamma n} \sum_{i=0}^{n}\left(\begin{array}{c}
m(n-2) \\
i
\end{array}\right)(\ln \xi)^{i} \Gamma((n-m-i), \alpha \Omega) .
\end{aligned}
$$

We know $\hat{F}(x)=\left\{1+(1 / t) \ln \left(1-e^{-(\gamma x)^{2}}\right)\right\}^{n-1}, \Omega<u<\infty$ and so we have

$$
\begin{aligned}
E[\hat{F}(x)]^{m} & =\int_{\Omega}^{\infty}\left[\{1+(1 / t) \ln \xi\}^{n-1}\right]^{m} \frac{\alpha^{n}}{\Gamma n} t^{n-1} e^{-\alpha t} d t \\
& =\frac{\alpha^{n}}{\Gamma n} \int_{\Omega}^{\infty} \sum_{i=0}^{m(n-1)}\left(\begin{array}{c}
m(n-1) \\
i
\end{array}\right)\left(\frac{\ln \xi}{t}\right)^{i} t^{n-1} e^{-\alpha t} d t \\
& =\frac{\alpha^{n}}{\Gamma n} \sum_{i=0}^{m(n-1)}\left(\begin{array}{c}
m(n-1) \\
i
\end{array}\right)(\ln \xi)^{i} \int_{\Omega}^{\infty} t^{n-i-1} e^{-\alpha t} d t \\
& =\frac{\alpha^{n}}{\Gamma n} \sum_{i=0}^{m(n-1)}\left(\begin{array}{c}
m(n-1) \\
i
\end{array}\right)(\ln \xi)^{i} \Gamma(n-i, \alpha \Omega),
\end{aligned}
$$

where $\Gamma(s, \alpha x)=\int_{x}^{\infty} t^{s-1} e^{-\alpha t} d t$ denotes the upper incomplete gamma function. 
Theorem 4. The mean squared error of estimator $\hat{f}(x)$ is given as

$$
\operatorname{MSE}(\hat{f}(x))=\left(\frac{(n-1) 2 x \eta}{\xi}\right)^{2} \frac{\alpha^{n}}{\Gamma n} \sum_{i=0}^{n}\left(\begin{array}{c}
2(n-2) \\
i
\end{array}\right)(\ln \xi)^{i} \Gamma((n-2-i), \alpha \Omega)-f^{2}(x)
$$

and the mean square error of estimator $\hat{F}(x)$ is given as

$$
\operatorname{MSE}(\hat{F}(x))=\frac{\alpha^{n}}{\Gamma n} \sum_{i=0}^{2(n-1)}\left(\begin{array}{c}
2(n-1) \\
i
\end{array}\right)(\ln \xi)^{i} \Gamma(n-i, \alpha \Omega)-F^{2}(x) .
$$

Proof. MSEs of estimators $\hat{f}(x)$ and $\hat{F}(x)$ are defined as

$$
\operatorname{MSE}(\hat{f}(x))=E(\hat{f}(x))^{2}-2 f(x) \hat{f}(x)+f^{2}(x)=E(\hat{f}(x))^{2}-f^{2}(x)
$$

and

$$
M S E(\hat{F}(x))=E(\hat{F}(x))^{2}-2 F(x) \hat{F}(x)+F^{2}(x)=E(\hat{F}(x))^{2}-F^{2}(x),
$$

respectively. Using equation (2.8) with $m=2$, we get the required MSE for $\hat{f}(x)$. Similarly we can obtain MSE of $\hat{F}(x)$ using equation (2.9) with $m=2$.

\subsection{Least Squares and Weighted Least Squares Estimators}

This section discusses about regression based estimators of unknown parameters. Swain et al. (1988) first suggested this method to estimate the parameters of beta distributions. Further many authors discussed and used this method for different distributions. Consider a random sample $X_{1}, \ldots, X_{n}$ of sample size $n$ from a $\operatorname{CDF} F(\cdot)$. Then we observe that

$E\left(F\left(X_{i}\right)\right)=\frac{i}{n+1}, V\left(F\left(X_{i}\right)\right)=\frac{i(n-i+1)}{(n+1)^{2}(n+2)}$, and $\operatorname{cov}\left[F\left(X_{i}\right), F\left(X_{j}\right)\right]=\frac{i(n-j-1)}{(n+1)^{2}(n+2)}$

for $i<j, i, j=1,2, \ldots, n$ (see, Johnson et al., 1994). Further we discuss two variants of this method namely least square and weighted least square estimators.

\subsubsection{Least Square Estimators (LSEs)}

In this method

$$
\sum_{i=1}^{n}\left[F\left(X_{i}\right)-\frac{i}{n+1}\right]^{2}
$$

is minimized with respect to the unknown parameters. For Burr $\mathrm{X}$ distribution, the expression

$$
\sum_{i=1}^{n}\left[\left(1-e^{-\left(\gamma x_{i}\right)^{2}}\right)^{\alpha}-\frac{i}{n+1}\right]^{2}
$$


is minimized with respect to the unknown shape parameter $\alpha$ (when $\gamma$ is known) and the least square estimator for $\alpha$ is denoted as $\hat{\alpha}_{l s}$.

Then we have

$$
\hat{f}_{l s}(x)=2 \hat{\alpha}_{l s} \gamma^{2} x e^{-(\gamma x)^{2}}\left(1-e^{-(\gamma x)^{2}}\right)^{\hat{\alpha}_{l s}-1} \text { and } \hat{F}_{l s}(x)=\left(1-e^{-(\gamma x)^{2}}\right)^{\hat{\alpha}_{l s}}
$$

as the least square estimators of the $f(x)$ and the $F(x)$, respectively. Further, simulation study is conducted to calculate the desired expectations and MSE values.

\subsubsection{Weighted Least Square Estimators (WLSEs)}

To obtain WLSE of the unknown parameters, we minimize the expression

$$
\sum_{i=1}^{n} w_{i}\left[F\left(x_{i}\right)-\frac{i}{n+1}\right]^{2}
$$

with respect to the unknown parameters. Here $w_{i}=\frac{1}{\operatorname{Var}\left[F_{X}\left(x_{i}\right)\right]}=\frac{(n+1)^{2}(n+2)}{i(n-i+1)}$ is defined as the weight function (see, Johnson et al., 1994). Note that the least square estimator is obtained under the consideration of constant variance. If such assumption does not hold true then weighted least square estimation may be considered with inverse of variance as weight. Under such scaling the corresponding error remains finite. For the Burr X distribution, the expression

$$
\sum_{i=1}^{n} \frac{(n+1)^{2}(n+2)}{i(n-i+1)}\left[\left(1-e^{-\left(\gamma x_{i}\right)^{2}}\right)^{\alpha}-\frac{i}{n+1}\right]^{2}
$$

is minimized with respect to the unknown shape parameter $\alpha$ (when $\gamma$ is known). Suppose $\hat{\alpha}_{w l s}$ denotes the WLSE of $\alpha$. Then we obtain the weighted LSEs of $f(x)$ and $F(x)$ as

$$
\hat{f}_{w l s}(x)=2 \hat{\alpha}_{w l s} \gamma^{2} x e^{\left.-(\gamma x)^{2}\right)}\left(1-e^{-(\gamma x)^{2}}\right)^{\hat{\alpha}_{w l s}-1} \text { and } \hat{F}_{w l s}(x)=\left(1-e^{-(\gamma x)^{2}}\right)^{\hat{\alpha}_{w l s}},
$$

respectively. Further we have conducted a simulation study to obtain the required expectations and MSE values.

\subsection{Estimators based on Percentiles}

This method was originally suggested by Kao $(1958,1959)$. A well explained explanations, on this topic, can be found in Mann et al. (1974); Johnson et al. (1994). Burr X distribution has closed form $\mathrm{CDF}$ and this method is based on inverting the CDF. So, estimation of parameters of this distribution can be done using this method.

Suppose $X_{1}, \ldots, X_{n}$ denotes an ordered random sample from Burr $\mathrm{X}$ distribution and $F\left(X_{i}\right)$ as the ordered distribution of the sample. Let $p_{i}=i /(n+1)$ then percentiles estimator of $\alpha$ denoted by $\hat{\alpha}_{p}$ is the one which minimizes the expression

$$
\sum_{i=1}^{n}\left[p_{i}-\left(1-e^{-\left(\gamma x_{i}\right)^{2}}\right)^{\alpha}\right]^{2} \text { or equivalently } \sum_{i=1}^{n}\left[\ln p_{i}-\alpha \ln \left(1-e^{-\left(\gamma x_{i}\right)^{2}}\right)\right]^{2}
$$


with respect to $\alpha$. Then

$$
\hat{f}_{p}(x)=2 \hat{\alpha}_{p} \gamma^{2} x e^{-(\gamma x)^{2}}\left(1-e^{-(\gamma x)^{2}}\right)^{\hat{\alpha}_{p}-1} \text { and } \hat{F}_{p}(x)=\left(1-e^{-(\gamma x)^{2}}\right)^{\hat{\alpha}_{p}}
$$

are the required percentile estimators of $f(x)$ and $F(x)$ respectively. Since it is difficult to find the expectations and the MSE values for these estimators analytically, so these can be obtained by means of simulations.

\subsection{Method of Maximum Product of Spacing}

The maximum product spacing (MPS) method has been introduced by Cheng and Amin (1979, 1983) as an alternative to MLE for the estimation of the unknown parameters parameters of continuous univariate distributions. Consider a sample of size $n$ be taken from a Burr X distribution. Then the corresponding uniform spacing is defined as

$$
D_{j}=F\left(x_{j}\right)-F\left(x_{j-1}\right), j=1,2, \ldots, n,
$$

where $F\left(x_{0: n}\right)=0, F\left(x_{n+1}\right)=1$ and $\sum_{j=1}^{n+1} D_{j}=1$. The MPS estimate of $\alpha$ denoted by $\hat{\alpha}_{m}$ is obtained by maximizing

$$
D(\alpha, \gamma)=\left[\prod_{j=1}^{n+1} D_{j}\right]^{\frac{1}{n+1}}
$$

with respect to the unknown shape parameter $\alpha$. Equivalently, the expression

$$
D^{*}(\alpha, \gamma)=\frac{1}{n+1} \sum_{j=1}^{n+1} \ln D_{j}
$$

can be maximized to obtain the estimate of $\alpha$ as desired. It can be shown that $\hat{\alpha}_{m}$ satisfies

$$
\frac{1}{n+1} \sum_{j=1}^{n+1} \frac{1}{D_{j}}\left(D_{0}\left(x_{j}\right)-D_{0}\left(x_{j-1}\right)\right)=0,
$$

where $D_{0}\left(x_{j}\right)=\left(1-e^{-\left(\gamma x_{j}\right)^{2}}\right)^{\alpha_{m}} \ln \left(1-e^{-\left(\gamma x_{j}\right)^{2}}\right)$. This has been shown by Cheng and Amin (1983) that the efficiency of MPS method of estimation is very close to the ML estimation method. Then $\hat{f}_{m}(x)$ and $\hat{F}_{m}(x)$ are the MPS estimators of $f(x)$ and $F(x)$ respectively and are given by

$$
\hat{f}_{m}(x)=2 \hat{\alpha}_{m} \gamma^{2} x e^{-(\gamma x)^{2}}\left(1-e^{-(\gamma x)^{2}}\right)^{\hat{\alpha}_{m}-1} \text { and } \hat{F}_{m}(x)=\left(1-e^{-(\gamma x)^{2}}\right)^{\hat{\alpha}_{m}},
$$

respectively. The expectations and the MSE of these estimators can be calculated using simulations. 


\subsection{Cramér-von-Mises Method of Estimation}

To motivate our choice of Cramér-von-Mises type minimum distance estimators, MacDonald (1971) provided empirical evidence that the bias of the estimator is smaller than the other minimum distance estimators. The value of $\alpha$ for which the function

$$
C(\alpha, \lambda)=\frac{1}{12 n}+\sum_{j=1}^{n}\left(F\left(x_{j}\right)-\frac{2 j-1}{2 n}\right)^{2}
$$

is minimized is defined as the Cramér-von-Mises estimator of $\alpha$ denoted by $\hat{\alpha}_{c}$. Equivalently, solution of the equation

$$
\sum_{j=1}^{n}\left(F\left(x_{j}\right)-\frac{2 j-1}{2 n}\right) D_{0}\left(x_{j}\right)=0
$$

gives the desired estimator $\hat{\alpha}_{c}$. Therefore Cramér-von-Mises estimators of $f(x)$ and $F(x)$ are $\hat{f}_{c}(x)$ and $\hat{F}_{c}(x)$ respectively and are given by

$$
\hat{f}_{c}(x)=2 \hat{\alpha}_{c} \gamma^{2} x e^{-(\gamma x)^{2}}\left(1-e^{-(\gamma x)^{2}}\right)^{\hat{\alpha}_{c}-1} \text { and } \hat{F}_{c}(x)=\left(1-e^{-(\gamma x)^{2}}\right)^{\hat{\alpha}_{c}}
$$

respectively. Simulations are used to obtain the expectations and the MSE values due to having difficulties in finding analytic solutions.

\subsection{Anderson-Darling Method of Estimation}

The Anderson-Darling test (Anderson and Darling, 1952) is as an alternative to other statistical tests for detecting sample distributions departure from normality. Specifically, the AD test converge very quickly towards the asymptote (Anderson and Darling, 1954; Pettitt, 1976; Stephens, 1974). The $\mathrm{AD}$ estimator $\hat{\alpha}_{a}$ of the unknown parameter $\alpha$ is obtained from the function

$$
A(\alpha, \gamma)=-n-\frac{1}{n} \sum_{j=1}^{n}(2 j-1)\left(\ln F\left(x_{j}\right)+\ln \bar{F}\left(x_{n+1-j}\right)\right),
$$

by minimizing with respect to $\alpha$. Equivalently, the solution of the equation

$$
\sum_{j=1}^{n}(2 j-1)\left(\frac{D_{0}\left(x_{j}\right)}{F\left(x_{j}\right)}-\frac{D_{0}\left(x_{n+1-j}\right)}{\bar{F}\left(x_{n+1-j}\right)}\right)=0,
$$

provides the desired estimator $\hat{\alpha}_{a}$ with $D_{0}(\cdot)$ being defined earlier. The Anderson- Darling estimators for $f(x)$ and $F(x)$ are presented as

$$
\hat{f}_{a}(x)=2 \hat{\alpha}_{a} \gamma^{2} x e^{-(\gamma x)^{2}}\left(1-e^{-(\gamma x)^{2}}\right)^{\hat{\alpha}_{a}-1} \text { and } \hat{F}_{a}(x)=\left(1-e^{-(\gamma x)^{2}}\right)^{\hat{\alpha}_{a}}
$$

respectively. The desired expectations and the MSE values of these estimators is difficult to find analytically. So simulations can be used. 


\section{Simulation Study}

We perform simulation study to compare the different estimators discussed here. We have arbitrarily considered four different sets parameter values, namely $(\alpha, \gamma)=(1,1),(1,2),(2,1),(2,2)$ to compare the performance of proposed methods. We mention that samples are generated from the Burr X distribution using the probability integral transformation method. We have computed results for arbitrarily selected sample sizes such as $n=10,20,30,40,50$. In fact deviation in MSEs of different estimators of PDF and CDF from the MSEs of corresponding ML estimator of PDF and $\mathrm{CDF}$ are obtained. Thus deviation of MSEs represent the difference between MSE of an estimator from the MSE of maximum likelihood estimator. We have computed these values for an arbitrary value $x=1$. The deviations of MSEs of different estimators are presented in Tables 1-2, from which we can easily say that MLEs are the most efficient estimators of the PDF and the CDF of a Burr X distribution and UMVUEs are the second most efficient estimators for the same. Since various deviations are positive hence we observe that ML estimators are having the lowest MSE values. Further visual analysis suggests that UMVUE has the second lowest MSE values for the PDF and CDF.

Table 1: Deviations of MSEs of the PDF for various methods from the MSEs of the PDF for MLE

\begin{tabular}{lllllllll}
\hline & & \multicolumn{7}{c}{ Deviations of MSEs of $f(x)$} \\
\cline { 3 - 9 } Para. & $n$ & UMVUE & LSE & WLSE & PCE & MPS & CVM & ADM \\
\hline$(1,1)$ & 10 & 0.00392 & 0.03284 & 0.01164 & 0.07164 & 0.00064 & 0.06621 & 0.01544 \\
& 20 & 0.00093 & 0.02971 & 0.01365 & 0.07635 & 0.00318 & 0.07387 & 0.01673 \\
& 30 & 0.00040 & 0.02707 & 0.01382 & 0.07767 & 0.00371 & 0.07389 & 0.01743 \\
& 40 & 0.00022 & 0.02409 & 0.01266 & 0.07927 & 0.00370 & 0.05940 & 0.01703 \\
& 50 & 0.00014 & 0.02121 & 0.01225 & 0.07518 & 0.00429 & 0.04295 & 0.01634 \\
& & & & & & & & \\
$(1,2)$ & 10 & $7.75 \times 10^{-04}$ & 0.08296 & 0.00319 & 0.00222 & 0.00239 & 0.43243 & 0.00075 \\
& 20 & $1.67 \times 10^{-04}$ & 0.03673 & 0.00123 & 0.00406 & 0.00154 & 0.30042 & 0.00067 \\
& 30 & $7.09 \times 10^{-05}$ & 0.01386 & 0.00072 & 0.00471 & 0.00143 & 0.24801 & 0.00106 \\
& 40 & $3.89 \times 10^{-05}$ & 0.00156 & 0.00051 & 0.00473 & 0.00127 & 0.19083 & 0.00117 \\
& 50 & $2.46 \times 10^{-05}$ & 0.00154 & 0.00039 & 0.00488 & 0.00125 & 0.13852 & 0.00123 \\
& & & & & & & & \\
$(2,1)$ & 10 & $8.94 \times 10^{-04}$ & 0.02566 & 0.04031 & 0.05968 & 0.00575 & 0.02778 & 0.00674 \\
& 20 & $2.04 \times 10^{-04}$ & 0.01529 & 0.03726 & 0.05318 & 0.00180 & 0.01617 & 0.00700 \\
& 30 & $6.94 \times 10^{-05}$ & 0.01198 & 0.03409 & 0.05142 & 0.00100 & 0.01218 & 0.00713 \\
& 40 & $3.11 \times 10^{-05}$ & 0.00945 & 0.03184 & 0.04974 & 0.00048 & 0.00954 & 0.00582 \\
& 50 & $1.65 \times 10^{-05}$ & 0.00868 & 0.03010 & 0.04664 & 0.00042 & 0.00874 & 0.00577 \\
& & & & & & & & \\
& & & & & & & & \\
& 10 & $2.67 \times 10^{-03}$ & 0.00472 & 0.01155 & 0.00765 & 0.01240 & 0.02978 & 0.00262 \\
& 20 & $5.79 \times 10^{-04}$ & 0.00617 & 0.00445 & 0.01568 & 0.00605 & 0.00848 & 0.00228 \\
& 30 & $2.43 \times 10^{-04}$ & 0.00596 & 0.00268 & 0.01711 & 0.00510 & 0.00603 & 0.00375 \\
& 40 & $1.33 \times 10^{-04}$ & 0.00632 & 0.00188 & 0.01813 & 0.00426 & 0.00636 & 0.00442 \\
& 50 & $8.57 \times 10^{-05}$ & 0.00608 & 0.00139 & 0.01904 & 0.00401 & 0.00609 & 0.00506 \\
\hline
\end{tabular}


Table 2: Deviations of MSEs of the CDF for various methods from the MSEs of the CDF for MLE

\begin{tabular}{|c|c|c|c|c|c|c|c|c|}
\hline \multirow[b]{2}{*}{ Para. } & \multirow[b]{2}{*}{$n$} & \multicolumn{7}{|c|}{ Deviations of MSEs of $F(x)$} \\
\hline & & UMVUE & LSE & WLSE & PCE & MPS & CVM & $\mathrm{ADM}$ \\
\hline \multirow[t]{5}{*}{$(1,1)$} & 10 & $6.1 \times 10^{-04}$ & 0.01866 & 0.01402 & 0.01784 & 0.00436 & 0.06226 & 0.00129 \\
\hline & 20 & $1.8 \times 10^{-04}$ & 0.01333 & 0.01611 & 0.02329 & 0.00469 & 0.05774 & 0.00464 \\
\hline & 30 & $8.4 \times 10^{-05}$ & 0.01107 & 0.01576 & 0.02495 & 0.00422 & 0.05217 & 0.00579 \\
\hline & 40 & $4.8 \times 10^{-05}$ & 0.00927 & 0.01422 & 0.02613 & 0.00363 & 0.03885 & 0.00609 \\
\hline & 50 & $3.1 \times 10^{-05}$ & 0.00774 & 0.01355 & 0.02520 & 0.00392 & 0.02616 & 0.00606 \\
\hline \multirow[t]{5}{*}{$(1,2)$} & 10 & $1.3 \times 10^{-05}$ & $1.2 \times 10^{-02}$ & $8.0 \times 10^{-05}$ & $3.3 \times 10^{-05}$ & $3.9 \times 10^{-05}$ & 0.05264 & $1.3 \times 10^{-05}$ \\
\hline & 20 & $2.8 \times 10^{-06}$ & $7.1 \times 10^{-03}$ & $5.7 \times 10^{-05}$ & $6.4 \times 10^{-05}$ & $2.5 \times 10^{-05}$ & 0.05896 & $1.0 \times 10^{-05}$ \\
\hline & 30 & $1.2 \times 10^{-06}$ & $4.4 \times 10^{-03}$ & $7.1 \times 10^{-05}$ & $7.7 \times 10^{-05}$ & $2.3 \times 10^{-05}$ & 0.06069 & $1.7 \times 10^{-05}$ \\
\hline & 40 & $6.4 \times 10^{-07}$ & $9.9 \times 10^{-04}$ & $5.4 \times 10^{-05}$ & $7.8 \times 10^{-05}$ & $2.1 \times 10^{-05}$ & 0.04678 & $1.8 \times 10^{-05}$ \\
\hline & 50 & $4.1 \times 10^{-07}$ & $2.4 \times 10^{-05}$ & $4.8 \times 10^{-05}$ & $7.7 \times 10^{-05}$ & $2.0 \times 10^{-05}$ & 0.03215 & $1.9 \times 10^{-05}$ \\
\hline \multirow[t]{5}{*}{$(2,1)$} & 10 & $1.1 \times 10^{-03}$ & 0.02161 & 0.03350 & 0.04983 & 0.00552 & 0.02177 & 0.00898 \\
\hline & 20 & $2.3 \times 10^{-04}$ & 0.01860 & 0.03204 & 0.05160 & 0.00531 & 0.01879 & 0.01025 \\
\hline & 30 & $9.5 \times 10^{-05}$ & 0.01765 & 0.03029 & 0.05409 & 0.00452 & 0.01781 & 0.01225 \\
\hline & 40 & $5.1 \times 10^{-05}$ & 0.01569 & 0.02888 & 0.05484 & 0.00449 & 0.01578 & 0.01171 \\
\hline & 50 & $3.2 \times 10^{-05}$ & 0.01563 & 0.02768 & 0.05391 & 0.00461 & 0.01569 & 0.01230 \\
\hline \multirow[t]{5}{*}{$(2,2)$} & 10 & $4.7 \times 10^{-05}$ & $1.0 \times 10^{-03}$ & 0.00022 & 0.00011 & $2.2 \times 10^{-04}$ & $2.5 \times 10^{-03}$ & $5.0 \times 10^{-05}$ \\
\hline & 20 & $1.0 \times 10^{-05}$ & $9.9 \times 10^{-05}$ & 0.00026 & 0.00025 & $1.0 \times 10^{-04}$ & $1.5 \times 10^{-04}$ & $3.5 \times 10^{-05}$ \\
\hline & 30 & $4.4 \times 10^{-06}$ & $9.6 \times 10^{-05}$ & 0.00023 & 0.00027 & $8.7 \times 10^{-05}$ & $9.7 \times 10^{-05}$ & $6.0 \times 10^{-05}$ \\
\hline & 40 & $2.4 \times 10^{-06}$ & $1.0 \times 10^{-04}$ & 0.00022 & 0.00029 & $7.2 \times 10^{-05}$ & $1.0 \times 10^{-04}$ & $7.1 \times 10^{-05}$ \\
\hline & 50 & $1.5 \times 10^{-06}$ & $9.9 \times 10^{-05}$ & 0.00026 & 0.00031 & $6.8 \times 10^{-05}$ & $9.9 \times 10^{-05}$ & $8.2 \times 10^{-05}$ \\
\hline
\end{tabular}

\section{Data Analysis}

In this section, we use a real data set to compare the performance of the suggested estimators of the PDF and CDF of Burr $X(\alpha, \gamma)$ distribution. The data set represent the number of cycles to failure for a group of 60 electrical items in a life test. The data was obtained from Lawless (2003, page 112).

Here, for computational ease, we have divided the whole data set by 1000 . The data set is fitted to Burr X distribution, generalized exponential distribution and generalized logistic distribution and for all these three distributions the estimates for parameters $\alpha$ and $\gamma$ together with KolmogrovSmirnov values and the p-values are calculated and presented in Table 3. It can be easily observed from the Kolmogrov-Simirnov and p-values that Burr X distribution fits the data better than the two competitors. Looking at the tabulated values in Table 4, we can conclude that the most efficient estimation method for fitting the data is the ML estimation method. Further different model section criteria namely maximum likelihood, Akaike information criterion, corrected Akaike information criterion, Bayes information criterion and Hannan-Quinn criterion defined by 
Table 3: Goodness of fit tests for proposed models in the real data set

\begin{tabular}{lllll}
\hline Distribution & $\alpha$ & $\gamma$ & KS & p-value \\
\hline Burr X & 0.362115 & 0.237414 & 0.062001 & 0.9642 \\
Generalized Logistic & 3.660288 & 0.809312 & 0.090870 & 0.6708 \\
Generalized Exponential & 0.915937 & 0.431173 & 0.092127 & 0.6544 \\
\hline
\end{tabular}

Table 4: Estimation of parameters and the model selection criteria for the real data set

\begin{tabular}{llllllll}
\hline Estimator & Estimate of $\alpha$ & Estimate of $\gamma$ & ML & AIC & AICc & BIC & HQC \\
\hline MLE & 0.362115 & 0.237414 & 210.3249 & 214.3249 & 214.5354 & 218.5136 & 215.9633 \\
LSE & 0.360121 & 0.229670 & 210.4142 & 214.4142 & 214.6247 & 218.6028 & 216.0526 \\
WLSE & 0.357896 & 0.232810 & 210.3522 & 214.3522 & 214.5627 & 218.5408 & 215.9906 \\
PCE & 0.321556 & 0.203874 & 211.8409 & 215.8409 & 216.0514 & 220.0296 & 217.4793 \\
MPS & 0.335072 & 0.219541 & 210.7966 & 214.7966 & 215.0072 & 218.9853 & 216.4351 \\
CVM & 0.371616 & 0.236671 & 210.3723 & 214.3723 & 214.5828 & 218.5610 & 216.0107 \\
AD & 0.359541 & 0.234239 & 210.3379 & 214.3379 & 214.5484 & 218.5266 & 215.9763 \\
\hline
\end{tabular}

$$
\begin{aligned}
\text { maximum likelihood }= & -2 \ln L(\theta), \\
\text { Akaike information criterion } & =-2 \ln L(\theta)+2 n_{p} \\
\text { Corrected Akaike information criterion } & =-2 \ln L(\theta)+2 n_{p}\left(\frac{n}{n-n_{p}-1}\right) \\
\text { Bayes information criterion } & =-2 \ln L(\theta)+n_{p} \ln (n) \text { and } \\
\text { Hannan-Quinn criterion } & =-2 \ln L(\theta)+2 n_{p} \ln (\ln (n)),
\end{aligned}
$$

respectively are used for assessing the behavior of the suggested methods of estimation. Here $\ln L(\theta)$ denotes the log-likelihood, $n$ denotes the number of observations in the data set, and $n_{p}$ denotes the number of parameters of the distribution. The smaller values of these model selection criteria leads to the better fit. It can easily be seen from Table 4 that the values of all the model selection criteria for ML estimation method are smaller than others. Thus the maximum likelihood method of estimation is preferred to use in practice.

\section{Conclusion}

In this article, we have considered eight methods of estimation of the probability density function and the cumulative distribution function for the $\operatorname{Burr} X(\alpha, \gamma)$ distribution and comparisons are performed. Such comparisons can be useful to find the best estimators for the PDF and the CDF which can be used to estimate functionals like differential entropy, Rényi entropy, Kullback-Leibler divergence, Fisher information, cumulative residual entropy, the quantile function, Bonferroni curve, Lorenz curve, probability weighted moments, hazard rate function, mean deviation about mean etc. From both simulation study and real data analysis, we observed that MLE performs better than their counter part. The performance of AD is fairly reasonable and competitive. Also, evidence based on the MSEs in the simulation study, the log-likelihood values, and the model selection criteria show that the ML estimators for the pdf and the CDF are the best. We hope our results and methods of 
estimation might attract wider sets of applications in the above mentioned functionals. As suggested by an anonymous reviewer, it would be interesting to investigate properties of different estimation methods of PDF and CDF under some censoring techniques as well, possibly using some different data sets. To the best of our knowledge, not much work has been done on this particular problem in literature. Also we have obtained results for considered estimation problem based on finite sample situations. More work is required to study the asymptotic behavior of such estimators. We will try to work on these aspects in near future.

\section{Acknowledgments}

The authors would like to thank a referee for careful reading and for valuable comments that greatly improved the article. They also thanks the Editor for constructive suggestions.

\section{References}

Ahmad, K., Fakhry, M., and Jaheen, Z. (1997), "Empirical Bayes estimation of $P(Y<X)$ and characterization of Burr-type X model," Journal of Statistical Planning and Inference, 64, 297 308.

Anderson, T. and Darling, D. (1952), "Asymptotic theory of certain 'goodness-of-fit' criteria based on stochastic processes," Annals of Mathematical Statistics, 23, 193-212.

— (1954), “A test of goodness of fit," Journal of American Statistical Association, 49, 765-769.

Asrabadi, B. (1990), "Estimation in the Pareto distribution," Metrika, 37, 199-205.

Aucoin, F., Ashkar, F., and Bayentin, L. (2012), "Parameter and quantile estimation of the 2parameter kappa distribution by maximum likelihood," Stochastic Environmental Research and Risk Assessment, 26, 1025-1039.

Bagheri, S., Alizadeh, M., and Nadarajah, S. (2014), "Efficient estimation of the PDF and the CDF of the Weibull extension model," Communications in Statistics - Simulation and Computation, DOI: 10.1080/03610918.2014.894059.

- (2016), "Efficient estimation of the PDF and the CDF of the exponentiated Gumbel distribution," Communications in Statistics-Simulation and Computation, 45: 339-361.

Bratpvrbajgyran, S. (2012), "A test of goodness of fit for Rayleigh distribution via cumulative residual entropy," Proceedings of the 8th World Congress in Probability and Statistics, 267-288.

Burr, I. (1942), "Cumulative frequency distribution," Annals of Mathematical Statistics, 13, 215231. 
Cheng, R. and Amin, N. (1979), "Maximum product of spacings estimation with application to the lognormal distribution," University of Wales Institute of Science and Technology, Cardiff, Math. Report, 79-1.

- (1983), "Estimating parameters in continuous univariate distributions with a shifted origin," Journal of Royal Statistical Society Series B, 45, 394-403.

Dattner, I. and Reiser, B. (2013), "Estimation of distribution functions in measurement error models," Journal of Statistical Planning and Inference, 143, 479-493.

Dixit, U. and Jabbari Nooghabi, M. (2010), "Efficient estimation in the Pareto distribution," Statistical Methodology, 7, 687-691.

- (2011), "Efficient estimation in the Pareto distribution with the presence of outliers," Statistical Methodology, 8, 340-355.

Durot, C., Huet, S., Koladjo, F., and Robin, S. (2013), "Least-squares estimation of a convex discrete distribution," Computational Statistics and Data Analysis, 67, 282-298.

Duval, C. (2013), "Density estimation for compound Poisson processes from discrete data," Stochastic Processes and Their Applications, 123, 3963-3986.

Er, G. (1998), "A method for multi-parameter PDF estimation of random variables," Structural Safety, 25-36.

Ferguson, T. S. (1967), "Mathematical Statistics: A Decision Theoretic Approach,” New York: Academic Press.

Hampel, D. (2008), "Estimation of differential entropy for positive random variables and its application in computational neuroscience," Modeling and Simulation in Science, Engineering and Technology, 213-224.

Jabbari Nooghabi, M. and Jabbari Nooghabi, H. (2010), "Efficient estimation of PDF, CDF and rth moment for the exponentiated Pareto distribution in the presence of outliers," Statistics, 44, 1-20.

Johnson, N., Kotz, S., and Balakrishnan, N. (1994), "Continuous Univariate Distribution," Vol. 1, 2nd edition, Wiley, New York.

Kao, J. (1958), "Computer methods for estimating Weibull parameters in reliability studies," Transaction of IRE-Reliability and Quality Control, 13, 15-22.

- (1959), "A graphical estimation of mixed Weibull parameters in life testing electron tubes," Technometrics, 1, 389-407.

Lawless, J. (2003), "Statistical Models and Methods for Lifetime Data," John Wiley and Sons, New York. 
Longford, N. T. (2012), "Small-sample estimators of the quantiles of the normal, log-normal and Pareto distributions," Journal of Statistical Computation and Simulation, 82, 1383-1395.

MacDonald, P. (1971), "Comment on a paper by Choi and Bulgren," Journal of Royal Statistical Society Series B, 33, 326-329.

Mann, N., Schafer, R., and Singpurwalla, N. (1974), "Methods for Statistical Analysis of Reliability and Life Data," .

Mielniczuk, J. and Wojtys, M. (2010), "Estimation of Fisher information using model selection," Metrika, 72, 163-187.

Nilsson, M. and Kleijn, W. B. (2007), "On the estimation of differential entropy from data located on embedded manifolds," IEEE Transactions on Information Theory, 53, 2330-2341.

Pettitt, A. (1976), "Cramer-von Mises statistics for testing normality with censored samples," Biometrika, 63, 475-481.

Przybilla, C., Fernandez-Canteli, A., and Castillo, E. (2013), "Maximum likelihood estimation for the three-parameter Weibull cdf of strength in presence of concurrent flaw populations," Journal of the European Ceramic Society, 33, 1721-1727.

Raqab, M. (1998), "Order statistics from the Burr Type X model," Computers Mathematics and Applications, 36, 111-120.

Raqab, M. and Kundu, D. (2006), "Burr type X distribution: revisited," Journal of Probability and Statistical Sciences, 4(2), 179-193.

Sartawi, H. and Abu-Salih, M. (1991), "Bayes prediction bounds for the Burr Type X model," Communication in Statistics - Theory and Methods, 20, 2307-2330.

Stephens, M. (1974), "EDF statistics for goodness of fit and some comparisons," Journal of American Statistical Association, 69, 730-737.

Surles, J. and Padgett, W. (1998), "Inference for $P(Y<X)$ in the Burr Type X model," Journal of Applied Statistical Science, 7, 225-238.

- (2001), "Inference for reliability and stress-strength for a scaled Burr Type X distribution," Lifetime Data Analysis, 7, 187-200.

- (2005), "Some Properties of a Scaled Burr type X Distribution," Journal of Statistical Planning and Inference, 128, 271-280.

Swain, J., Venkatraman, S., and Wilson, J. (1988), "Least squares estimation of distribution function in Johnson's translation system," Journal of Statistical Computation and Simulation, 29, 71-297.

Received: January 26, 2018

Accepted: July 17, 2018 